\title{
A Low Dose of Dietary Resveratrol Partially Mimics Caloric Restriction and Retards Aging Parameters in Mice
}

\author{
Jamie L. Barger ${ }^{1}$, Tsuyoshi Kayo ${ }^{1}$, James M. Vann ${ }^{2,3}$, Edward B. Arias ${ }^{4}$, Jelai Wang ${ }^{5}$, Timothy A. Hacker ${ }^{6}$, \\ Ying Wang ${ }^{7}$, Daniel Raederstorff ${ }^{7}$, Jason D. Morrow ${ }^{8,9}$, Christiaan Leeuwenburgh ${ }^{10}$, David B. Allison ${ }^{11}$, \\ Kurt W. Saupe ${ }^{6}$, Gregory D. Cartee ${ }^{4}$, Richard Weindruch ${ }^{12 *}$, Tomas A. Prolla ${ }^{2,3 *}$
}

1 LifeGen Technologies, LLC, Madison, Wisconsin, United States of America, 2 Department of Medical Genetics, University of Wisconsin, Madison, Wisconsin, United States of America, 3 Department of Genetics, University of Wisconsin, Madison, Wisconsin, United States of America, 4 Division of Kinesiology, University of Michigan, Ann Arbor, Michigan, United States of America, $\mathbf{5}$ Section on Statistical Genetics, Department of Biostatistics, University of Alabama at Birmingham, Birmingham, Alabama, United States of America, 6 Department of Medicine, University of Wisconsin-Madison, Madison, Wisconsin, United States of America, 7 R\&D Human Nutrition and Health, DSM Nutritional Products Ltd., Basel, Switzerland, 8 Department of Pharmacology, Vanderbilt University School of Medicine, Nashville, Tennessee, United States of America, 9 Department of Medicine, Vanderbilt University School of Medicine, Nashville, Tennessee, United States of America, 10 Department of Aging and Geriatrics and College of Medicine, University of Florida, Gainesville, Florida, United States of America, 11 Section on Statistical Genetics, Department of Biostatistics and Clinical Nutrition Research Center, University of Alabama at Birmingham, Birmingham, Alabama, United States of America, 12 Department of Medicine and Veterans Administration Hospital, University of Wisconsin-Madison, Madison, Wisconsin, United States of America

\begin{abstract}
Resveratrol in high doses has been shown to extend lifespan in some studies in invertebrates and to prevent early mortality in mice fed a high-fat diet. We fed mice from middle age (14-months) to old age (30-months) either a control diet, a low dose of resveratrol $\left(4.9 \mathrm{mg} \mathrm{kg}^{-1} \mathrm{day}^{-1}\right)$, or a calorie restricted (CR) diet and examined genome-wide transcriptional profiles. We report a striking transcriptional overlap of $\mathrm{CR}$ and resveratrol in heart, skeletal muscle and brain. Both dietary interventions inhibit gene expression profiles associated with cardiac and skeletal muscle aging, and prevent age-related cardiac dysfunction. Dietary resveratrol also mimics the effects of $C R$ in insulin mediated glucose uptake in muscle. Gene expression profiling suggests that both CR and resveratrol may retard some aspects of aging through alterations in chromatin structure and transcription. Resveratrol, at doses that can be readily achieved in humans, fulfills the definition of a dietary compound that mimics some aspects of CR.
\end{abstract}

Citation: Barger JL, Kayo T, Vann JM, Arias EB, Wang J, et al. (2008) A Low Dose of Dietary Resveratrol Partially Mimics Caloric Restriction and Retards Aging Parameters in Mice. PLoS ONE 3(6): e2264. doi:10.1371/journal.pone.0002264

Editor: Daniel Tomé, AgroParisTech, France

Received February 12, 2008; Accepted April 20, 2008; Published June 4, 2008

Copyright: $\odot 2008$ Barger et al. This is an open-access article distributed under the terms of the Creative Commons Attribution License, which permits unrestricted use, distribution, and reproduction in any medium, provided the original author and source are credited.

Funding: CL: NIH AG17994 and AG21042, DBA: NSF EPSCoR 05026, KAS: NIH AG-000908, JDM: NIH GM15431, DK48831 and ES13125, TAP: NIH AG020681 Portions of this research were funded by DSM Nutritional Products, Ltd.; however, DSM had no role in study design, data collection and analysis, decision to publish, or preparation of the manuscript.

Competing Interests: T.A. Prolla and R. Weindruch are founders and board directors of LifeGen Technologies, LLC, a company focused on the use of gene expression profiling to understand the mechanisms of action of caloric restriction.

*E-mail: rhweindr@wisc.edu (RW); taprolla@wisc.edu (TP)

\section{Introduction}

Caloric restriction $(\mathrm{CR})$ retards several aspects of the aging process in mammals, including age-related mortality, tumorigenesis, physiological decline [1] and the establishment of age-related transcriptional profiles [2]. The wide scope of these actions, and the profound metabolic and hormonal shifts induced by GR has led to efforts at identifying natural or synthetic compounds that mimic the effects of $\mathrm{CR}$ in the absence of overt metabolic and endocrine disturbances or reduced caloric intake. Because most age-related diseases are likely to be secondary to the aging process itself, the discovery of such compounds could have a profound public health impact by reducing disease incidence and possibly extending the quality and length of the human lifespan.

Resveratrol, a natural compound found in grapes and red wine has previously been shown to extend lifespan in $S$. cerevisiae, $C$. elegans and Drosophila through a SIRT1 dependent mechanism $[3,4]$. However, recent studies have failed to reproduce these life extension results $[5,6]$, and other studies have demonstrated that the ability of resveratrol to activate yeast Sir2 or human SIRT1 is substrate-specific in vitro [7] and resveratrol has no effect on Sir2 activity in vivo [5]. Thus, the effects and mechanisms of resveratrol in life extension in invertebrates are currently unclear. Recently, mice fed a high fat diet supplemented with high levels of resveratrol (22 or $186 \mathrm{mg} \mathrm{kg}^{-1} \mathrm{day}^{-1}$ ) were shown to have extended lifespan as compared to controls, and several metabolic alterations similar to what is observed with CR, including markers of increased mitochondrial biogenesis [8,9]. Because control animals in this study had early mortality due to toxicity of the high fat diet, the role of resveratrol in the aging process in mammals is also unclear and a detailed examination of the physiological impact of resveratrol on aging and health parameters is indicated.

Global gene expression profiling can be used to evaluate the biological age of a tissue, because as shown for animals subjected to $\mathrm{CR}$, such profiles correlate with biological as opposed to chronological age [2]. Given that compounds that retard aging may do so in a tissue-specific manner, we and others have used 
gene expression profiling as a method to search for compounds that can mimic the effects of CR [10,11]. This study aimed to examine the role of resveratrol in gene expression profiles associated with mammalian aging and the specific effects of both resveratrol and GR on gene expression patterns in multiple tissues. We also compared the effects of resveratrol and CR in insulin signaling, oxidative damage, age-related cardiac function, and spontaneous tumorigenesis.

\section{Results}

\section{Global impact of CR and resveratrol on age-related changes in gene expression}

We fed individually-housed male $(\mathrm{C} 57 \mathrm{BL} / 6 \times \mathrm{C} 3 \mathrm{H} / \mathrm{He}) \mathrm{F}_{1}$ hybrid mice one of three diet formulations: a control diet $(84$ $\mathrm{kcal}_{\text {mouse }}^{-1}$ week $\left.^{-1}\right)$, a CR diet $\left(63 \mathrm{kcal} \mathrm{mouse}^{-1}\right.$ week $\left.^{-1}\right)$ and a control diet supplemented with trans-resveratrol $\left(4.9 \mathrm{mg} \mathrm{kg}^{-1}\right.$ day $^{-1}$ ) starting at middle age (14 months of age). We have previously reported that this CR regimen started at middle age in this strain leads to a $13 \%$ increase in average and maximum lifespan [10]. As expected, long-term feeding of the CR diet resulted in a significant reduction in body weight (for control mice, average body weight $35 \mathrm{~g}$; for CR mice average body weight $24 \mathrm{~g} ; P<0.01$, Supplemental Figure $\mathrm{S} 1$ ); this reduction in body weight was not observed in resveratrol-fed animals (Supplemental Figure S1). Mice were sacrificed at 30-31 months of age and tissues collected for gene expression analysis. We focused our analysis on three tissues: heart, skeletal muscle and brain (neocortex).

To examine the age-associated changes in gene expression we compared transcriptional profiles of young and old mice fed the control diet. There were 1,029 genes $(P \leq 0.01)$ that were significantly changed in expression with age in the heart. We next examined the impact of $\mathrm{CR}$ on the expression of these genes by comparing 30-month old CR mice and 30-month old control mice. Age-related changes in gene expression that are prevented by $\mathrm{CR}$ would be expected to show opposite directions of change (upregulation vs. downregulation). As previously reported [2], we observed a large effect of CR in opposing age-related changes: CR reduced $921(90 \%)$ age-related alterations in gene expression and 536 of these represented highly significant differences $(P \leq 0.01)$ in expression between the old control and old CR groups (Figure 1A). To examine the effect of resveratrol supplementation on agerelated gene expression patterns, we performed a similar analysis comparing 30-month old control mice and 30-month old resveratrol fed animals (Figure 1A). Surprisingly, resveratrol opposed $947(92 \%)$ of age-related changes in gene expression, and 522 of these represented highly significant differences in expression between the old control and old resveratrol groups $(P \leq 0.01)$. Thus, resveratrol at doses that can be readily achieved through dietary supplementation in humans is as effective as CR in opposing the majority of age-related transcriptional alterations in the aging heart. Because the collection of such alterations in gene expression is a biomarker of aging, our results imply that similar to $\mathrm{CR}$, middle-age onset resveratrol supplementation at low doses is likely a robust intervention in the retardation of cardiac aging. Lesser effects on aging inhibition by $\mathrm{CR}$ and resveratrol were obtained in skeletal muscle; aging resulted in alteration of expression of 515 skeletal muscle genes, $136(26 \%)$ of which were significantly $(P \leq 0.01)$ opposed by $\mathrm{CR}$ and resveratrol (Figure 1A). In neocortex, CR and resveratrol significantly inhibited only 19 and $13 \%$ respectively of the 505 highly significant age-related changes in gene expression (Figure 1A).

\section{$\mathrm{CR}$ and resveratrol have overlapping effects on alterations in gene expression in multiple tissues}

We next examined the transcriptional profiles of CR and resveratrol fed mice for genes that are impacted by the dietary interventions, but are not altered in expression with aging. Such changes in gene expression represent shifts induced by the dietary interventions and can provide clues to mechanisms of action and the degree and nature of CR mimicry by resveratrol. We identified 747 genes that were significantly altered in expression by both CR and resveratrol in the heart $(P \leq 0.01)$ but were not altered in expression as a result of normal aging. Strikingly, 745 (99.7\%) of these gene expression alterations occurred in the same direction for both treatments (Figure 1B). This finding was also observed in muscle (1164/1164 genes, Figure 1B) and brain (1129/1134 genes, Figure 1B). Clearly, resveratrol can mimic a large component of the transcriptional profile of $\mathrm{CR}$ in all tissues examined.

\section{Resveratrol and CR prevent age-related cardiac dysfunction}

Cardiac function is known to decline with age in mice and humans, a factor that likely contributes to the fact that cardiac disease is one of the leading contributors to age-related disability and death. To assess left ventricular (LV) systolic and diastolic function as well as structure, we used in vivo M-mode and Doppler echocardiography to examine cardiac function in young (five month-old) control mice and 25 month-old control, GR and resveratrol fed mice. Isovolumic relaxation time, a measure of diastolic function, was increased in aged animals (Figure 2A), consistent with the impaired LV relaxation that occurs with normal aging in rodents and humans [12]. Both CR and resveratrol supplementation reduced the age-related increase in this parameter, though these changes were not statistically significant. We also examined the myocardial performance index, a parameter that provides an overall assessment of cardiac function [13]. An increased index value is associated with reduced cardiac performance, and this value increased significantly with age (Figure 2A). Both CR and resveratrol supplementation almost completely prevented the age-related decrease in this parameter. Thus, resveratrol mimics the effects of $\mathrm{CR}$ to prevent cardiac aging at both the transcriptional and functional levels.

\section{Effects of resveratrol and CR on endocrine status and glucose metabolism}

In an attempt to uncover the mechanisms of action of resveratrol in retarding aging parameters and its ability to mimic $\mathrm{CR}$, we examined pathways implicated in aging and CR. In mammals, spontaneous mutations that result in growth hormone (GH) deficiency, such as the Prop- 1 and Pit-1 dwarf mice $[14,15]$, or targeted mutations in the insulin-like growth factor 1 (IGF-1) receptor gene [16] or the insulin receptor substrate 1 (IRS1) can result in increased lifespan [17], suggesting an important role for IGF-1 in longevity. Some of the phenotypes that are observed in animals with altered IGF-1 or insulin signaling are also observed in GR mice, such as reduced levels of IGF-1, insulin and glucose [18]. To investigate if resveratrol inhibits transcriptional profiles of aging and mimics CR through an endocrine mechanism, we measured glucose, T3, insulin, IGF-1 and GH in five month-old control, CR and resveratrol fed mice. Following two months of dietary intervention, we observed reduced IGF-1 levels in CR mice, but not in resveratrol treated mice (Figure 2B). We did not observe significant alterations in any other hormones examined. Surprisingly, because we observed a large overlap of transcriptional shifts induced by resveratrol and CR in all organs examined, 


\section{A. PREVENTION OF AGE-RELATED CHANGES IN GENE EXPRESSION}

\section{HEART}
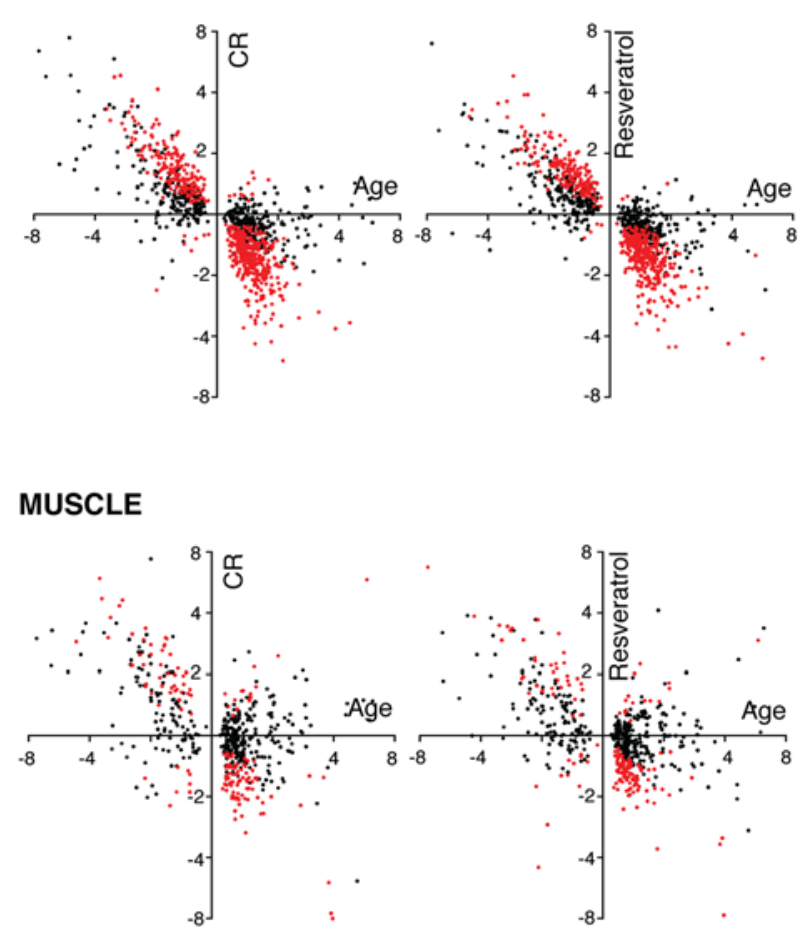

\section{BRAIN}

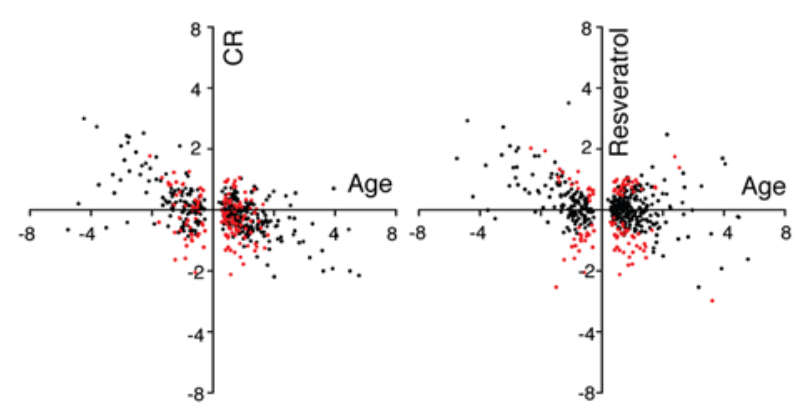

\section{B. TREATMENT-INDUCED CHANGES IN GENE EXPRESSION}

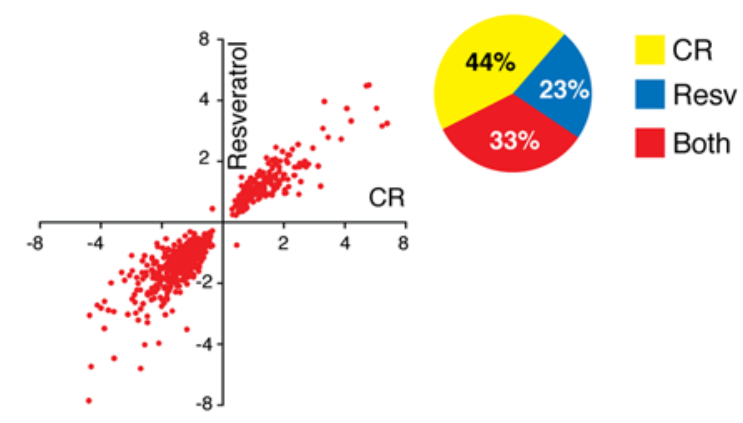

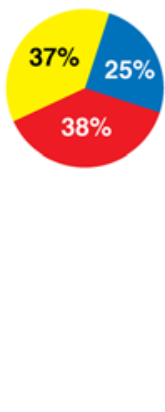
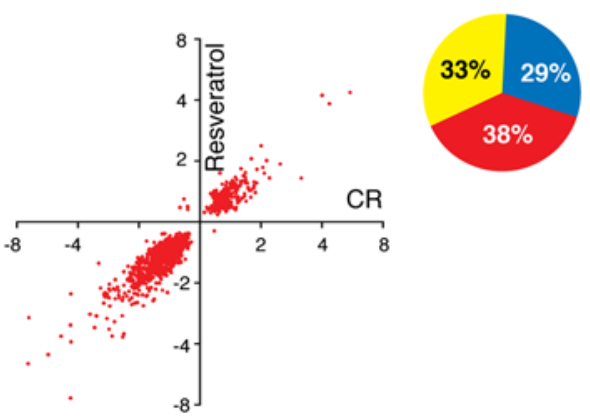

Figure 1. Global effects of resveratrol and CR on gene expression. Gene expression profiling of 20,687 unique transcripts using Affymetrix Mouse Genome 4302.0 arrays was performed in young (5-months old) control and old control, CR and resveratrol fed animals (30-months old, all groups). (A). A panel of genes corresponding to significant $(P \leq 0.01)$ changes in gene expression in the comparison between young and old control groups was examined in both CR (left) and resveratrol fed mice (right). Numbers on $X$ and $Y$ axes represent fold changes in the young vs. old comparison ( $\mathrm{X}$ axis), and the old $\mathrm{CR}$ vs old control ( $\mathrm{Y}$ axis, left graphs) or old resveratrol vs. old control group ( $\mathrm{Y}$ axis, right graphs). Each dot corresponds to an specific gene. Red dots correspond to genes that are significantly altered in expression at $P \leq 0.01$ in both aging and $C R$, or aging and resveratrol comparisons. Opposite fold changes in this analysis represent prevention of aging changes. (B). A panel of genes significantly changed by $C R$ and resveratrol (but not by age) as compared to old control mice $(P \leq 0.01)$ is plotted. Similar fold changes in this analysis represent resveratrol mimicry of CR. Pie charts represent the proportion of genes changed by CR only, resveratrol only or both treatments. Gene expression changes plotted in graphs correspond to the genes changed significantly expression by both treatments (red).

doi:10.1371/journal.pone.0002264.g001

our findings also suggest that a large component of the transcriptional program induced by CR may be independent of CR-mediated alterations in plasma IGF-1, or insulin. This conclusion is supported by the finding that dwarfism and CR may impact lifespan through independent mechanisms [19], and the finding that GH deficiency and CR display minimal overlap at the gene expression level [20].
Genetic alteration of insulin related pathways has clearly been implicated in aging retardation in multiple organisms, and earlier studies have demonstrated that mice consuming a CR diet [21] or supplemented with resveratrol have improved insulin sensitivity $[8,9]$. Both CR and resveratrol supplementation lowered blood glucose levels ( $P=0.06$ for CR, $P=0.07$ for resveratrol; Figure 2B). Neither diet lowered plasma insulin (data not shown). We next 

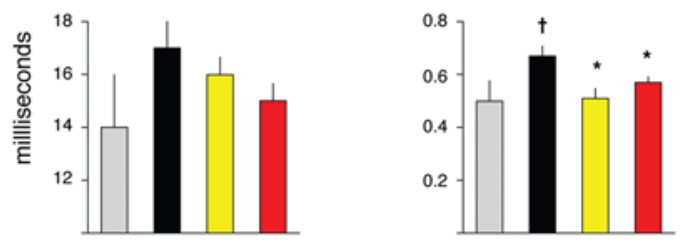

Young Control

Old Control

$\mathrm{CR}$

Resv

B

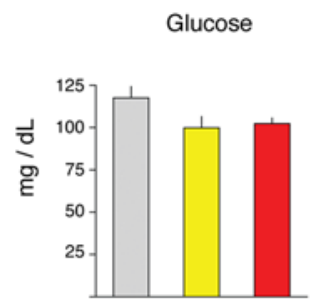

Plasma IGF-1

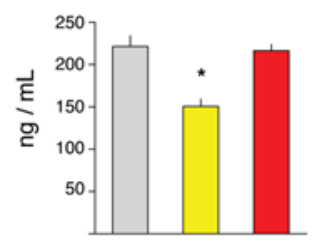

C
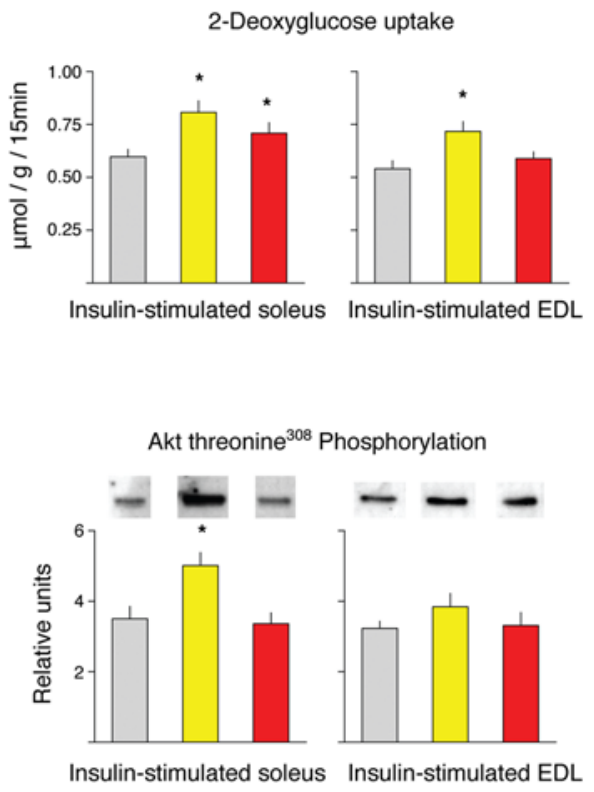

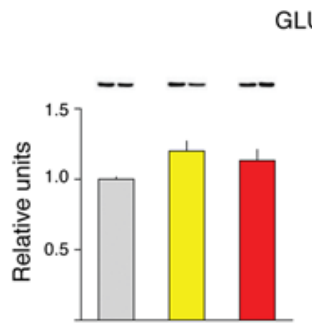

Soleus
GLUT4

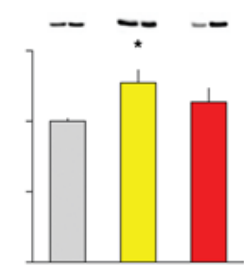

EDL

Figure 2. Effects of $C R$ and resveratrol on physiological parameters. (A) Cardiac function as determined by transthoracic echocardiography $(n=7, Y C, n=10$ OC, $n=8$ CR, $n=9$ Resv) (B) Serum glucose and plasma IGF-1 in control, CR and resveratrol treated mice (C) Glucose uptake and insulin signaling in control, $C R$ and resveratrol fed animals $(n=16)$. Rate of 2-deoxyglucose uptake and Akt threonine ${ }^{308}\left(T^{308}\right)$ phosphorylation were determined in isolated paired soleus and extensor digitorum longus (EDL) muscles with or without $0.36 \mathrm{nM}$ insulin. Data were analyzed by two-way ANOVA, and the source of significant variance was tested using Student-Newman-Keuls post-hoc test ( $\mathrm{n}=15-16$ muscles per group). There were no differences between groups at baseline so values shown reflect insulin-stimulated values only. GLUT4 protein abundance was determined in soleus and EDL muscles by western blotting ( $\mathrm{n}=16$ muscles per group). Data were analyzed by one-way ANOVA on ranks, and the source of significant variance was tested using Dunn's post-hoc test.

doi:10.1371/journal.pone.0002264.g002

examined 2-deoxyglucose (2-DG) uptake in isolated soleus and extensor digitorum longus (EDL) muscles from mice fed CR or resveratrol diets from three to five months of age. CR resulted in a significant increase in 2-DG uptake in the insulin-stimulated soleus $(35 \%)$ and EDL (32\%) muscles, without any effect on 2-DG uptake in the basal condition. There was also an increase in 2-DG uptake in the insulin-stimulated soleus (18\%), but not in the EDL, of resveratrol treated mice. Akt phosphorylation, which partially mediates enhanced insulin sensitivity of muscle [22], was increased with GR but not with resveratrol supplementation (Figure 2G). Levels of GLUT4, the major glucose transporter in muscle, were also increased in CR, but not resveratrol-treated mice (Figure 2B). These findings suggest that the mechanism of the increased glucose uptake in insulin-stimulated skeletal muscle in resveratrol treated mice is different from that of CR mice, as neither Akt phosphorylation nor GLUT4 content were elevated by resveratrol. 
CR and resveratrol do not alter SIRT1 levels, and CR but not resveratrol induces PGC- $1 \alpha$ transcriptional targets

Feeding high levels of resveratrol to mice has been shown to be associated with increased SIRT 1 activity as measured by PGC- $1 \alpha$ acetylation and induction of its transcriptional targets $[8,9]$. Because sirtuin overexpression increases longevity in some organisms and may mediate some of the effects of CR [3,4], the induction of SIRT1 activity has been postulated to mediate the health benefits of resveratrol $[8,9]$. We investigated if alterations in the levels of SIRT1 or induction of PGC- $1 \alpha$ transcriptional activity can explain the observation that a low dose of resveratrol mimics CR. In contrast to previous finding in rats [23] and humans [24], neither CR nor resveratrol feeding significantly altered the levels of SIRT1 protein in brain or liver of mice, and SIRT1 abundance was actually significantly decreased in both heart and muscle of CR mice (Figure 3A). However, CR clearly increased the mRNA levels of Pgc- $1 \alpha$ in skeletal muscle. GR also stimulated an increase in Pgc- $1 \alpha$ transcriptional targets Pdk4 and Ucp3 in heart and skeletal muscle. In contrast, resveratrol did not significantly increase the levels of Pgc- $1 \alpha$ expression or any of its transcriptional targets, with the exception of a small effect on the expression of Pdk4 in skeletal muscle (Figure 3B). Thus, our microarray results suggest that a low dose of dietary resveratrol induces a transcriptional program similar to $\mathrm{CR}$ in multiple tissues and retards aging parameters, but these effects may be largely independent of the increase in SIRT1 activity and activation of Pgc- $1 \alpha$ transcriptional targets reported previously for mice in a high fat diet fed high levels of resveratrol $[8,9]$.

\section{Effects of $C R$ and resveratrol feeding on oxidative stress parameters}

Because resveratrol has been shown to act as an antioxidant both in vitro and in vivo, we tested the hypothesis that resveratrol supplementation retards aging through a reduction in oxidative damage which has been postulated to be causal in both aging and a number of age-related diseases. To test if resveratrol has an impact on spontaneous oxidative damage, we measured F2isoprostanes, a marker of lipid peroxidation in heart, skeletal muscle and brain of mice fed CR or resveratrol diets from three to five months of age (Supplemental Figure 2). Surprisingly, we found that resveratrol significantly raised the levels of F2-isoprostanes in the heart relative to controls $(P<0.05)$ and isoprostane levels in the brain were higher in resveratrol treated mice compared CR mice $(P<0.01)$; isoprostane levels were similar among groups in muscle. We next examined oxidative damage to DNA and RNA in the same mice; levels of 8-hydroxy-2'-deoxyguanosine (8-OHdG) and 8-hydroxyguanosine (8-OHG) were similar among groups for each tissue (Supplemental Figure S2). Thus, our observations do not indicate lowering of spontaneous levels of oxidative damage by either CR or resveratrol.

\section{Functional analysis of $C R$ and resveratrol-mediated gene expression changes in multiple tissues}

Because resveratrol did not impact well-known factors that are postulated to impact aging (IGF-1, insulin, Sirt1, oxidative stress), we analyzed the gene expression data for functional categories that may be affected with age, CR or resveratrol. We used the significance analysis of function and expression (SAFE), a twostage, permutation-based method that can be applied to various experimental designs, accounts for the unknown correlation among genes and enables permutation-based estimation of error rates [25]. We used the Gene Ontology (GO) Biological Process classification scheme for annotating gene function and only included GO terms that were represented by least 10 genes. This resulted in 571 GO terms represented in each tissue (Supplemental Table S1). Because our global analysis of age-independent shifts in gene expression indicated that resveratrol mimics CR in all tissues examined, we were particularly interested in functional classes that were significantly altered by both interventions across tissues. Only four GO terms were impacted by both GR and resveratrol across all tissues (Figure 4), and these were chromatin assembly or disassembly (GO:0006333), regulation of transcription from RNA Polymerase II promoter (GO:0006357), transcription from RNA polymerse II promoter (GO:0006366), and ubiquitin cycle (GO:0006512). Analysis of individual genes within these classes suggests that both $\mathrm{CR}$ and resveratrol have a major impact on expression of genes that play important roles in chromatin remodeling that defines the transcriptional/epigenetic state of the cell. In skeletal muscle (Figure 5), these include the de novo methylase Dnmt3a, the chromodomain helicase DNA binding protein 1 (Chd1), the sirtuin Sirt5, and the SWI/SNF complex members Smarcc1, Smarca4, Smarca5 and Smarca2. The Smarca2 gene encodes Brahma, the key component of the SWI/SNF complex, a master transcriptional switch that directs specific cellular programs and uses ATP hydrolysis to remodel chromatin [26]. Additionally, multiple histone encoding genes were altered in expression by both CR and resveratrol. Alteration in expression of the same or related genes was also observed in heart and brain.

\section{Discussion}

The importance of chromatin remodeling proteins in defining the chromatin state of the cell, basal transcription and their ability to influence DNA repair [27] suggests a common mechanism of action for $\mathrm{CR}$ and resveratrol. Based on the functional analysis of gene expression profiles in multiple tissues we postulate that both CR and resveratrol impact pathways that determine chromatin remodeling, perhaps in response to a metabolic stress signal. The ensuing alteration in chromosome architecture and transcription may facilitate pathways that maintain genomic stability, or prevent epigenetic alterations, and therefore retard some aspects of the aging process in the long-term. Understanding the pathways that influence the expression of genes involved in chromatin remodeling and transcription in response to both $\mathrm{CR}$ and resveratrol may therefore provide key insights into the molecular basis of aging in mammals.

Our studies suggest that dietary consumption of a low dose of resveratrol partially mimics $\mathrm{CR}$ and inhibits some aspects of the aging process. In long lived rodent strains and in humans, lifespan is often limited by spontaneous tumorigenesis. Studies have determined that the ability of CR to inhibit spontaneous tumorigenesis is linked to the CR-mediated reduction in circulating IGF-1 [28], and in the case of mammary carcinogenesis can be reversed by the administration of IGF-1 to CR animals [29]. Our study design involved the use of a long-lived F1 hybrid mouse strain, and sacrificing mice at 30-months of age, therefore we were unable to evaluate effects of resveratrol on average or maximum lifespan. We note that unlike CR, resveratrol did not reduce circulating IGF-1 levels (Figure 2B), and there was also no decrease in spontaneous tumors at the time of sacrifice (Supplemental Table S2). In particular, spontaneous liver tumors were abundant in mice fed the control diet or resveratrol, but rare in CR mice. Thus, although a low dose of resveratrol can improve quality of life by retarding aging parameters such as cardiac dysfunction, a nutritional or pharmaceutical strategy to also 
A

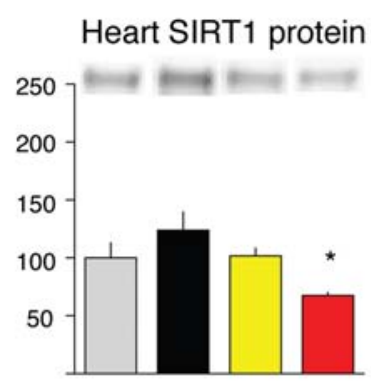

Muscle SIRT1 protein

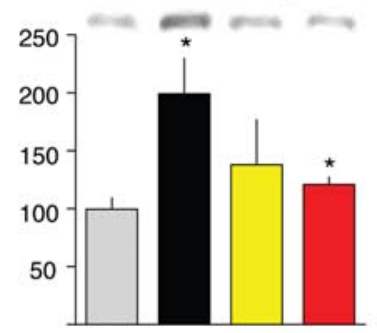

Brain SIRT1 protein

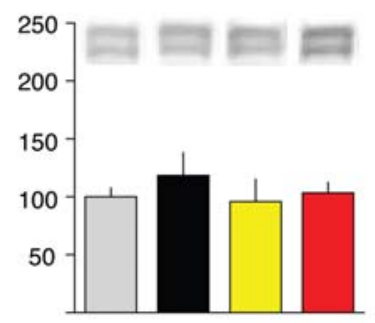

Liver SIRT1 protein

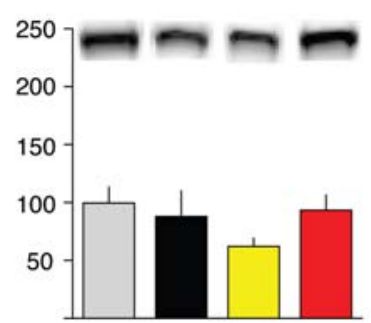

B

Pgc-1a

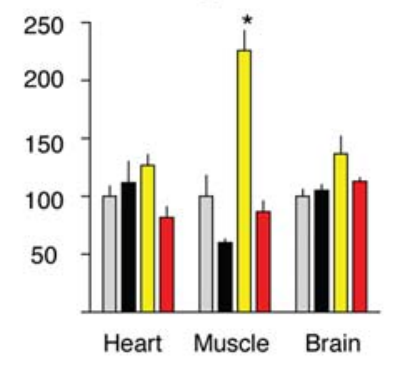

Young Control

Old Control

$\mathrm{CR}$

Resv
Pdk4

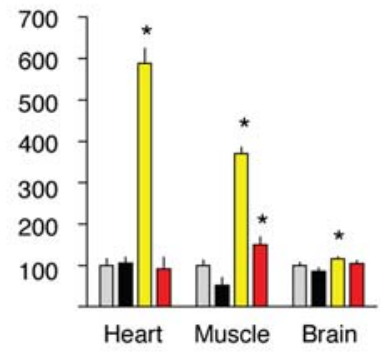

Ucp3

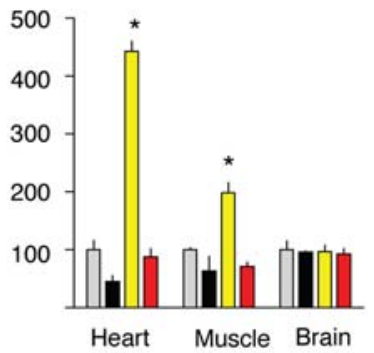

Nrf1

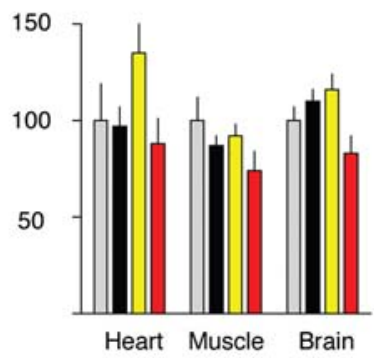

Tfam

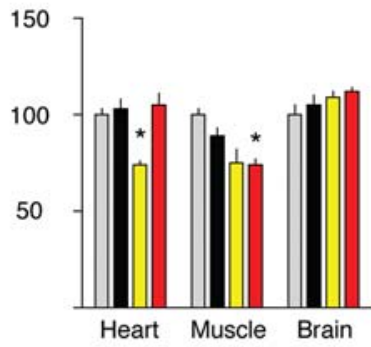

Figure 3. SIRT1 levels and Pgc1- $\alpha$ transcriptional activity in response to CR and resveratrol. (A) SIRT1 levels in liver, skeletal muscle and brain $(n=$ at least four animals per group) were determined by Western blot analysis. A loading correction factor based on HSP70 band intensity data was used to normalize the SIRT1 band intensity data. (B) mRNA abundance for known Pgc-1 $\alpha$ transcriptional targets is shown for heart, muscle and brain. Data on $\mathrm{Y}$ axis represent percentage changes relative to young controls. Results represent $n=5$, values in bar graphs are means and SE. ${ }^{*} p<0.01$.

doi:10.1371/journal.pone.0002264.g003

increase lifespan in mice will likely require blockage of the IGF-1 axis or its targets.

Our study also raises questions regarding proposed mechanisms of action of both CR and resveratrol. Findings from previous studies performed with higher doses of resveratrol in mice suggested that an increase in SIRT1 activity and the resulting deacetylation of the transcriptional coactivator Pgc- $1 \alpha$ is a central mechanism of action. Health benefits observed included reduced mortality associated with the high-fat diet, improved motor performance and improved insulin sensitivity [8,9]. Surprisingly, 


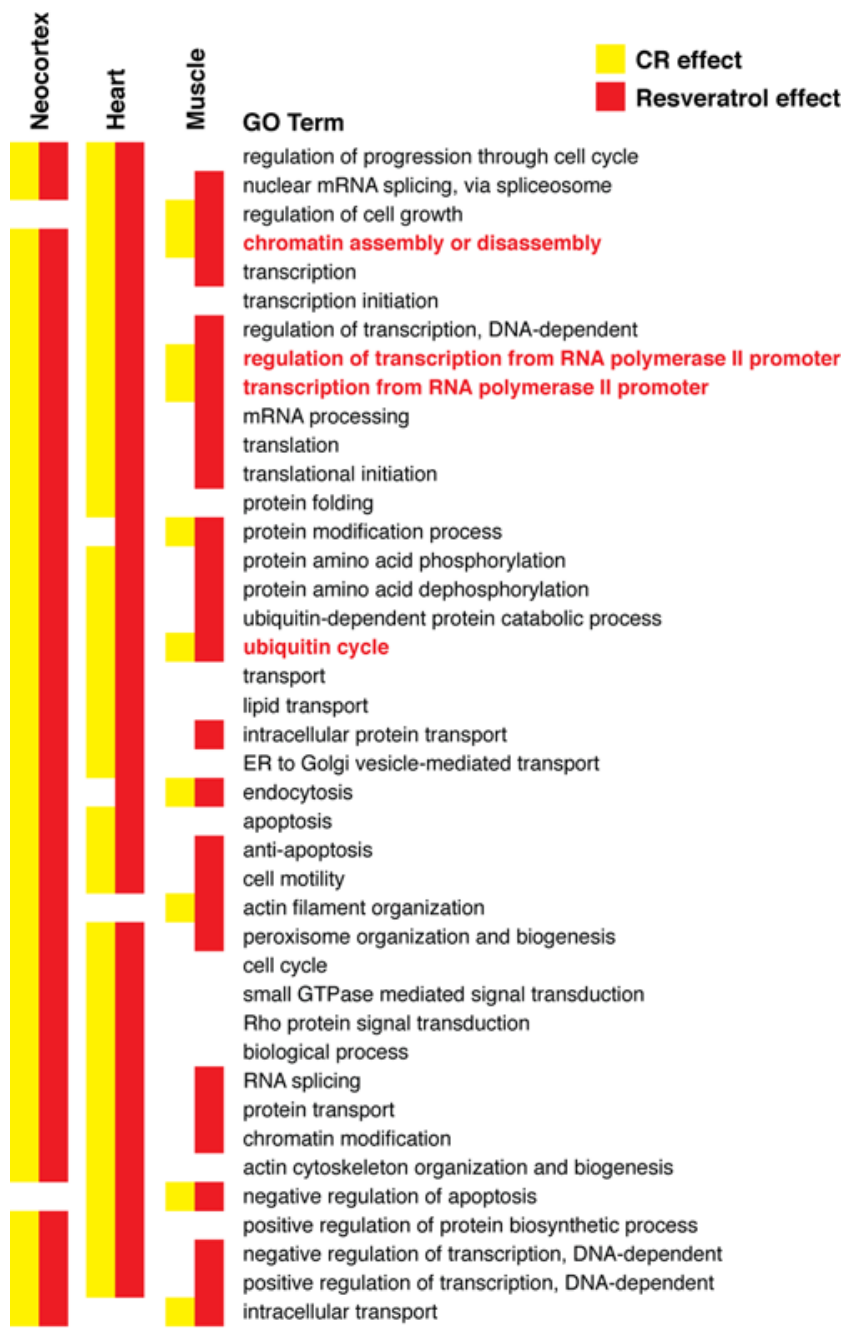

Figure 4. Functional gene expression analysis of $C R$ and resveratrol fed mice using SAFE. A class matrix, which describes the functional categories and specifies what genes are members of what classes, was based on Gene Ontology (GO), and included classes with at least 10 genes, for a total of 571 classes. The SAFE analysis was then run using the default settings for the local statistic (Student's $t$ ) and GO terms that differed at $\mathrm{P} \leq 0.05$ were considered significantly different. Only classes that show a significant effect for at least one treatment in one tissue are shown. Significance values for all functional classes are shown in Supplemental Table S1. Classes highlighted in blue were changed by both $\mathrm{CR}$ and resveratrol in all tissues examined. doi:10.1371/journal.pone.0002264.g004

the induction of Pgc- $1 \alpha$ transcriptional targets was observed in skeletal muscle but not heart [9]. Given that resveratrol is known to be cardioprotective in models of ischemia-reperfusion [30], and our own findings of strong activity in the retardation of cardiac aging, but no effects on Pgc- $1 \alpha$ transcriptional targets, it seems unlikely that SIRT1/Pgc- $1 \alpha$ play a role in resveratrol's cardiac effects. It is likely that the effects of resveratrol feeding at lower doses reported here are distinct than those observed with higher doses, with particular relevance to the induction of SIRT1 activity. We also did not find support for the hypothesis that induction of SIRT1 directly mediates the effects of GR in the tissues examined, since SIRT1 levels were not altered at the mRNA or protein levels. Previous studies in rats [23] and humans [24] suggest that CR induces SIRT1 in these species, but the only study in mice that addressed this issue used an "every other day" feeding protocol, and therefore does not represent CR [31]. To our knowledge our study is the first to attempt to detect an induction of SIRT1 in CR mice, and we have not observed such induction in any of the tissues examined (heart, liver, skeletal muscle and brain). Given that overexpression of SIRT1 in mice does induce physiological alterations consistent with CR [32], it is possible that GR impacts the levels of SIRT1 and other sirtuins in other tissues that play a central role in regulating metabolism, such the pancreas [33,34]. We also note that despite the absence of SIRT1 induction, our findings are consistent with a general alteration in the expression of genes involved in chromatin remodeling by CR and resveratrol, including other sirtuins (Figure 5). Other proposed biochemical mechanisms of action of resveratrol that were not examined in this study may include stimulation of AMP kinase [35], and increased nitric oxide synthase activity [36-38]. We also note that studies suggest that the nutrient sensor target of rapamycin (TOR) pathway, involved in the regulation of growth and autophagy mediates the life-extension effects of GR in Drosophila [39] and C. elegans [40], and therefore may play a role in the resveratrol effects reported here. Because resveratrol mimics $\mathrm{CR}$ at the gene expression level, but did not mimic the effects of CR on the few proteins examined in this study (GLUT4, AKT, IGF-1 and SIRT1) it is possible that although similar at the transcriptional level, CR and resveratrol have different effects with regard to translational regulation. An examination of several proteins encoded by genes affected in expression by both $\mathrm{CR}$ and resveratrol should clarify this issue.

Our findings that a low dose of resveratrol partially mimics CR at the gene expression level and leads to prevention of some agerelated parameters suggests that clinical trials with resveratrol should be conducted to test the relevance of these findings to humans. Because cardiac disease is a major contributor to agerelated mortality, positive findings could lead to a novel and important approach to improve the quality of human life.

\section{Materials and Methods}

\section{Animals and diets}

Male $\mathrm{C} 57 \mathrm{BL} / 6 \times \mathrm{C} 3 \mathrm{H} / \mathrm{He} \mathrm{F} 1$ hybrid mice were purchased at six weeks of age (Harlan-Teklad), individually housed in a specific pathogen free facility and fed a control diet based on the AIN-93M formulation (Bio-Serv, Frenchtown, NJ). Upon arrival and until 14 months of age, mice received $84 \mathrm{kcal}$ per week, approximately $10 \%$ lower than ad lib intake. Starting at 14 months of age, mice were randomly divided into one of three groups: a Control group receiving the same diet; a resveratrol-treated group receiving the same diet as the controls but supplemented with $50 \mathrm{mg}$ resveratrol per $\mathrm{kg}$ diet (resveratrol purchased from Sigma Chemical, St. Louis); this dose is approximately equal to $4.9 \mathrm{mg}$ resveratrol per $\mathrm{kg}$ body weight assuming a $35 \mathrm{~g}$ mouse; a calorie restricted (CR) group receiving $63 \mathrm{kcal}$ per week. Details on feeding Control and CR diets have been published elsewhere [41]. At 30 months of age, tissues were collected from mice, flash-frozen in liquid nitrogen and stored at $-80^{\circ} \mathrm{C}$. Tissues were also collected from a group of young control mice that received the control diet from two to five months of age. The number of spontaneous deaths in each cohort prior to the age of sacrifice was 2/30,0/18 and 2/22 in the control, CR and resveratrol-treated groups, respectively (Supplemental Table S2). Studies in young mice were performed as described above except the CR or resveratrol treatments were begun at two months of age and were continued through five months of age. All procedures complied with the Institutional Animal Care and Use Committee of the William S. Middleton Memorial Veterans Hospital, Madison, WI 


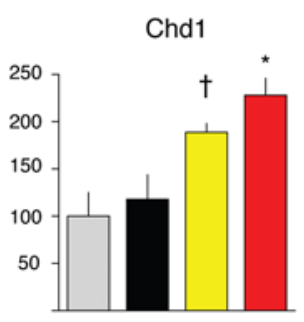

H3ł3a

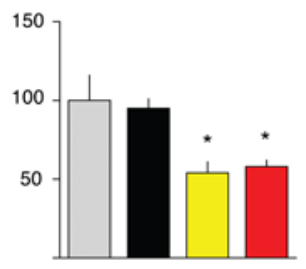

Sirt2

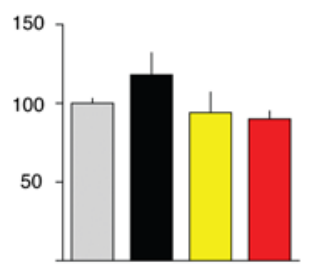

Dnmt3a

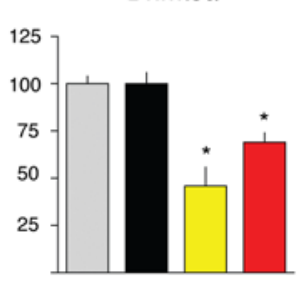

Hist1h2bc

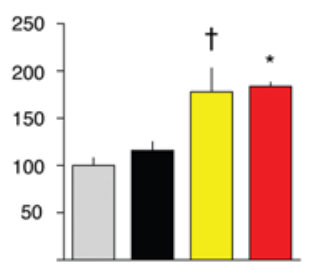

Sirt3

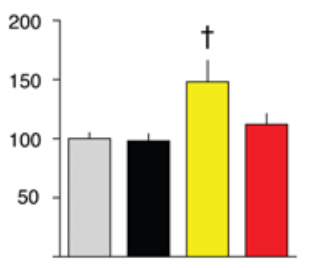

Young Control

Old Control

CR

Resv

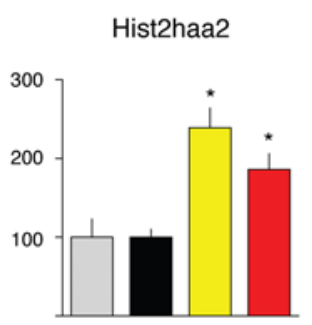

Sirt5

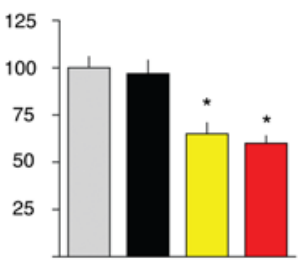

Smarca2

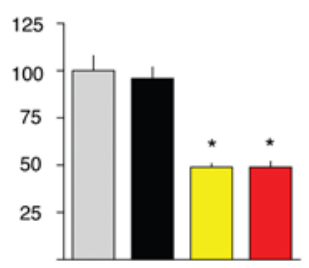

Smarca4

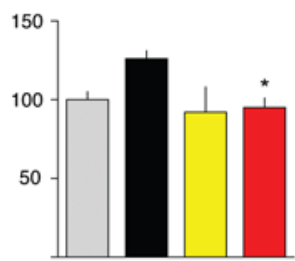

Smarca5

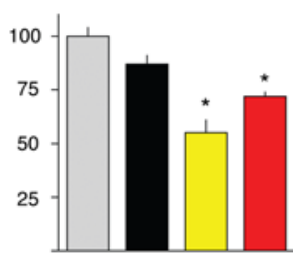

Smarcc1

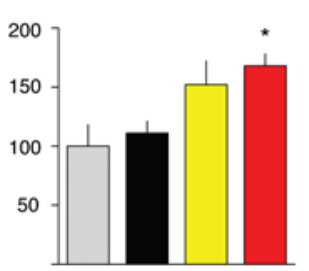

Figure 5. Selected genes in skeletal muscle associated with GO:0006333 (chromatin assembly and disassembly). Means identified with $\dagger$ were significantly different from age-matched controls at $p<0.05$; means identified with * were significantly different from age-matched controls at $\mathrm{p}<0.01$.

doi:10.1371/journal.pone.0002264.g005

\section{Gene expression profiling/Functional Classification}

Gene expression profiling was performed using Affymetrix Mouse Genome 4302.0 arrays as described previously [2]. Aging comparisons were made between Young and Old Control groups; $\mathrm{CR}$ and resveratrol effects were determined by comparing treated mice to the Old Control group. Genes were considered to be significantly different at $P \leq 0.01$ using Student's $t$-tests.

To discover gene classes that are significantly different between the two groups in each comparison, we used the Significance Analysis of Function and Expression (SAFE) analysis [25]. This approach is a two-stage, permutation-based method that can be applied to various experimental designs, accounts for the unknown correlation among genes and enables permutation-based estimation of error rates. The SAFE analysis requires three input data structures: the gene expression data, a response vector, and a class matrix. The first two were created from the array data using custom source code to reformat the data to conform to what SAFE expects. The class matrix, which describes the functional categories and specifies what genes are members of what classes, was created in a similar way and was based on Gene Ontology (GO), and included classes with at least 10 genes. This resulted in 571 classes. The SAFE analysis was then run using the default settings for the local statistic (Student's $t$ ) and GO terms that differed at $\mathrm{P} \leq 0.05$ were considered significantly different.

\section{Cardiac function}

Transthoracic echocardiography was performed using an Acuson Sequoia (Siemens) ultrasonograph with a $15-\mathrm{MHz}$ transducer. For acquisition of two-dimensional guided M-mode images at the tips of papillary muscles and Doppler studies, mice were sedated by IP administration of $100 \mathrm{mg} / \mathrm{kg}$ ketamine and maintained on a heated platform in a left lateral decubitus position. The chest was shaved and prewarmed coupling gel applied. Transmitral velocities were measured using Doppler pulse 
wave imaging. All images were saved to an on-board optical disk. Echocardiograms were performed and analyzed by an investigator blinded to the experimental groups.

End diastolic and systolic left ventricular (LV) diameter as well as anterior and posterior wall (AW and PW respectively) thicknesses were measured on line from M-mode images using the leading edge-to-leading edge convention. All parameters were measured over at least three consecutive cardiac cycles and averaged. Left ventricular fractional shortening was calculated as $\left[\left(\mathrm{LV}\right.\right.$ diameter $_{\text {diastole }}-\mathrm{LV}$ diameter $\left._{\text {systole }}\right) / \mathrm{LV}$ diameter $\left._{\text {diastole }}\right] \times$ 100 and LV mass was calculated by using the formula. Relative wall thickness was calculated as $2 \times$ Posterior wall diastole $_{\text {l }} / \mathrm{LV}$

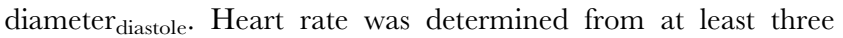
consecutive intervals from the pulse wave Doppler tracings of the LV outflow tract. Isovolumic relaxation time was measured as the time from the closing of the aortic value to the opening of the mitral value from pulse wave Doppler tracings of the LV outflow tract and mitral inflow region. Myocardial Performance Index $(\mathrm{MPI})$ was calculated as $(\mathrm{a}-\mathrm{b}) / \mathrm{b}$ where $\mathrm{a}=$ mitral valve closure time and $b=$ aortic injection time

\section{Glucose uptake and insulin signaling}

Rate of 2-deoxyglucose uptake and Akt threonine ${ }^{308}\left(\mathrm{~T}^{308}\right)$ phosphorylation were determined in isolated paired soleus and extensor digitorum longus (EDL) muscles at baseline with or without $0.36 \mathrm{nM}$ insulin. Data were analyzed by two-Way ANOVA, and the source of significant variance was tested using Student-Newman-Keuls post-hoc test $(n=15-16$ muscles per group). There were no differences between groups at without insulin so values shown reflect insulin-stimulated values only.

GLUT4 protein abundance was determined in soleus and EDL muscles by western blotting ( $\mathrm{n}=16$ muscles per group). Data were analyzed by one-way ANOVA on ranks, and the source of significant variance was tested using Dunn's post-hoc test.

\section{SIRT1 abundance}

Samples for western blotting were electrophoresed in polyacrylamide tris-acetate gels (Invitrogen) and transferred to nitrocellulose. Membranes were blocked in $0.5 \%$ gelatin in TBS-T for one hour. Primary and secondary antibodies were diluted in $0.5 \%$ gelatin in TBS-T. Rabbit anti-SIRT1 was purchased from Upstate (Charlottesville, VA), goat anti-HSP70 and horseradish peroxidase (HRP)-linked donkey anti-goat IgG were purchased from Santa Cruz Biotechnology (Santa Cruz, CA). HRP-linked goat antirabbit IgG was purchased from Pierce (Rockford, IL). Chemiluminescent bands were visualized and analyzed using a UVP

\section{References}

1. Weindruch R, Walford RL (1988) The Retardation of Aging and Disease By Dietary Restriction. Springfield, Ill.: Charles C Thomas. 436 p.

2. Lee CK, Klopp RG, Weindruch R, Prolla TA (1999) Gene expression profile of aging and its retardation by caloric restriction. Science 285: 13901393 .

3. Howitz KT, Bitterman KJ, Cohen HY, Lamming DW, Lavu S, et al. (2003) Small molecule activators of sirtuins extend Saccharomyces cerevisiae lifespan. Nature 425: 191-196.

4. Wood JG, Rogina B, Lavu S, Howitz K, Helfand SL, et al. (2004) Sirtuin activators mimic caloric restriction and delay ageing in metazoans. Nature 430: 686-689.

5. Kaeberlein M, McDonagh T, Heltweg B, Hixon J, Westman EA, et al. (2005) Substrate-specific activation of sirtuins by resveratrol. J Biol Chem 280: 17038-17045.

6. Bass TM, Weinkove D, Houthoofd K, Gems D, Partridge L (2007) Effects of resveratrol on lifespan in Drosophila melanogaster and Caenorhabditis elegans. Mech Ageing Dev 128: 546-552.

7. Borra MT, Smith BC, Denu JM (2005) Mechanism of human SIRT1 activation by resveratrol. J Biol Chem 280: 17187-17195.
Bioimaging Systems (Upland, CA) EpiChemi II and LabWorks Image Acquisition and Analysis Software. HSP70 was used as a loading control. A loading correction factor based on the HSP70 band intensity data was used to adjust the SIRT1 band intensity data.

\section{Isoprostanes/nucleic acid damage}

Isoprostanes were quantified in skeletal muscle tissue utilizing a highly precise and accurate method employing stable isotope dilution mass spectrometry as described previously [42].

RNA and DNA were simultaneously isolated from tissues by homogenization in 3M guanidine isothiocyanate (GTC) followed by a phenol-chloroform extraction procedure with inclusion of the metal chelator desferoxamine. Samples were hydrolyzed using nuclease $\mathrm{P}_{1}$ and alkaline phosphatase into single nucleosides and analyzed using a highly sensitive HPLC/EC/UV system (Coulochem III, ESA Inc., Chelmsford, MA). Samples were compared against a calibration curve with known standards to quantify the levels of the oxidative products 8-hydroxy-2'-guanosine $/ 10^{6} 2^{\prime}$ guanosine (RNA) or 8-hydroxy-2' -deoxyguanosine $/ 10^{6} 2^{\prime}$-deoxyguanosine (DNA).

\section{Supporting Information}

Table S1 Complete list of Gene Ontology Biological Processes from the SAFE analysis

Found at: doi:10.1371/journal.pone.0002264.s001 (0.11 MB PDF)

Table S2 Necropsy results from old mice fed a control, GR or resveratrol-supplemented diet

Found at: doi:10.1371/journal.pone.0002264.s002 (0.04 MB PDF)

\section{Acknowledgments}

The authors wish to acknowledge the assistance of Deepak Kini for testing and quality control with the SAFE analysis. The authors also wish to thank Maria Seabra and Tim Hofer for technical assistance with the nucleic acid damage assays.

\section{Author Contributions}

Conceived and designed the experiments: TP RW. Performed the experiments: TK JV EA TH. Analyzed the data: TP JW RW JB. Contributed reagents/materials/analysis tools: DA JW GC CL KS YW DR JM. Wrote the paper: TP RW JB.

8. Baur JA, Pearson KJ, Price NL, Jamieson HA, Lerin C, et al. (2006) Resveratrol improves health and survival of mice on a high-calorie diet. Nature 444: 337-342.

9. Lagouge M, Argmann C, Gerhart-Hines Z, Meziane H, Lerin C, et al. (2006) Resveratrol improves mitochondrial function and protects against metabolic disease by activating SIRT1 and PGG-1alpha. Cell 127: 1109-1122.

10. Lee CK, Pugh TD, Klopp RG, Edwards J, Allison DB, et al. (2004) The impact of alpha-lipoic acid, coenzyme Q10 and caloric restriction on life span and gene expression patterns in mice. Free Radic Biol Med 36: 1043-1057.

11. Dhahbi JM, Mote PL, Fahy GM, Spindler SR (2005) Identification of potential caloric restriction mimetics by microarray profiling. Physiol Genomics 23: 343-350.

12. Lakatta EG, Sollott SJ (2002) Perspectives on mammalian cardiovascular aging: humans to molecules. Comp Biochem Physiol A Mol Integr Physiol 132: 699-721.

13. Broberg CS, Pantely GA, Barber BJ, Mack GK, Lee K, et al. (2003) Validation of the myocardial performance index by echocardiography in mice: a noninvasive measure of left ventricular function. J Am Soc Echocardiogr 16: 814-823. 
14. Flurkey K, Papaconstantinou J, Harrison DE (2002) The Snell dwarf mutation Pitl(dw) can increase life span in mice. Mech Ageing Dev 123: 121-130.

15. Brown-Borg HM, Borg KE, Meliska CJ, Bartke A (1996) Dwarf mice and the ageing process. Nature 384: 33.

16. Holzenberger M, Dupont J, Ducos B, Leneuve P, Geloen A, et al. (2003) IGF-1 receptor regulates lifespan and resistance to oxidative stress in mice. Nature 421: 182-187.

17. Selman C, Lingard S, Choudhury AI, Batterham RL, Claret M, et al. (2007) Evidence for lifespan extension and delayed age-related biomarkers in insulin receptor substrate 1 null mice. FASEB J.

18. Longo VD, Finch CE (2003) Evolutionary medicine: from dwarf model systems to healthy centenarians? Science 299: 1342-1346.

19. Bartke A, Wright JC, Mattison JA, Ingram DK, Miller RA, et al. (2001) Extending the lifespan of long-lived mice. Nature 414: 412

20. Miller RA, Chang Y, Galecki AT, Al-Regaiey K, KopchickJJ, et al. (2002) Gene expression patterns in calorically restricted mice: partial overlap with long-lived mutant mice. Mol Endocrinol 16: 2657-2666.

21. Gazdag AC, Dumke CL, Kahn CR, Cartee GD (1999) Calorie restriction increases insulin-stimulated glucose transport in skeletal muscle from IRS-1 knockout mice. Diabetes 48: 1930-1936.

22. McCurdy CE, Cartee GD (2005) Akt2 is essential for the full effect of calorie restriction on insulin-stimulated glucose uptake in skeletal muscle. Diabetes 54: 1349-1356.

23. Cohen HY, Miller C, Bitterman KJ, Wall NR, Hekking B, et al. (2004) Calorie restriction promotes mammalian cell survival by inducing the SIRT1 deacetylase. Science 305: 390-392.

24. Civitarese AE, Carling S, Heilbronn LK, Hulver MH, Ukropcova B, et al. (2007) Calorie restriction increases muscle mitochondrial biogenesis in healthy humans. PLoS Med 4: e76.

25. Barry WT, Nobel AB, Wright FA (2005) Significance analysis of functional categories in gene expression studies: a structured permutation approach. Bioinformatics 21: 1943-1949.

26. Sengupta S, Xiong L, Fathalli F, Benkelfat C, Tabbane K, et al. (2006) Association study of the trinucleotide repeat polymorphism within SMARCA2 and schizophrenia. BMC Genet 7: 34

27. Osley MA, Tsukuda T, Nickoloff JA (2007) ATP-dependent chromatin remodeling factors and DNA damage repair. Mutat Res 618: 65-80.

28. Hursting SD, Switzer BR, French JE, Kari FW (1993) The growth hormone: insulin-like growth factor 1 axis is a mediator of diet restriction-induced inhibition of mononuclear cell leukemia in Fischer rats. Cancer Res 53: 2750-2757.

29. Dunn SE, Kari FW, French J, Leininger JR, Travlos G, et al. (1997) Dietary restriction reduces insulin-like growth factor I levels, which modulates apoptosis, cell proliferation, and tumor progression in p53-deficient mice. Cancer Res 57: 4667-4672.

30. Das DK, Maulik N (2006) Resveratrol in cardioprotection: a therapeutic promise of alternative medicine. Mol Interv 6: 36-47.

31. Nisoli E, Tonello C, Cardile A, Cozzi V, Bracale R, et al. (2005) Calorie restriction promotes mitochondrial biogenesis by inducing the expression of eNOS. Science 310: 314-317

32. Bordone L, Cohen D, Robinson A, Motta MC, van Veen E, et al. (2007) SIRT1 transgenic mice show phenotypes resembling calorie restriction. Aging Cell 6: 759-767.

33. Bordone L, Motta MC, Picard F, Robinson A, Jhala US, et al. (2006) Sirt1 regulates insulin secretion by repressing UCP2 in pancreatic beta cells. PLoS Biol 4: e31.

34. Haigis MC, Mostoslavsky R, Haigis KM, Fahie K, Christodoulou DC, et al. (2006) SIRT4 inhibits glutamate dehydrogenase and opposes the effects of calorie restriction in pancreatic beta cells. Cell 126: 941-954.

35. Dasgupta B, Milbrandt J (2007) Resveratrol stimulates AMP kinase activity in neurons. Proc Natl Acad Sci U S A 104: 7217-7222.

36. Klinge CM, Wickramasinghe NS, Ivanova MM, Dougherty SM (2008) Resveratrol stimulates nitric oxide production by increasing estrogen receptor \{alpha\}-Src-caveolin-1 interaction and phosphorylation in human umbilical vein endothelial cells. FASEB J.

37. Penumathsa SV, Thirunavukkarasu M, Zhan L, Maulik G, Menon VP, et al. 2008) Resveratrol enhances GLUT-4 translocation to the caveolar lipid raft fractions through AMPK/AKT/eNOS signaling pathway in diabetic myocardium. J Cell Mol Med.

38. Thirunavukkarasu M, Penumathsa SV, Koneru S, Juhasz B, Zhan L, et al (2007) Resveratrol alleviates cardiac dysfunction in streptozotocin-induced diabetes: Role of nitric oxide, thioredoxin, and heme oxygenase. Free Radic Biol Med 43: 720-729.

39. Kapahi P, Zid BM, Harper T, Koslover D, Sapin V, et al. (2004) Regulation of lifespan in Drosophila by modulation of genes in the TOR signaling pathway. Curr Biol 14: 885-890.

40. Hansen M, Taubert S, Crawford D, Libina N, Lee SJ, et al. (2007) Lifespan extension by conditions that inhibit translation in Caenorhabditis elegans. Aging Cell 6: 95-110.

41. Pugh TD, Klopp RG, Weindruch R (1999) Controlling caloric consumption: protocols for rodents and rhesus monkeys. Neurobiol Aging 20: 157-165.

42. Morrow JD, Roberts LJ 2nd (1999) Mass spectrometric quantification of F2isoprostanes in biological fluids and tissues as measure of oxidant stress. Methods Enzymol 300: 3-12. 